\title{
PONDx: real-life utilization and decision impact of the 21-gene assay on clinical practice in Italy
}

Francesco Cognetti ${ }^{1 凶}$, Riccardo Masetti ${ }^{2}$, Alessandra Fabi ${ }^{3}$, Giulia Bianchi ${ }^{4}$, Donatella Santini ${ }^{5}$, Alessia Rognone ${ }^{6}$, Giovanna Catania $^{3}$, Domenico Angelucci ${ }^{7}$, Giuseppe Naso ${ }^{8}$, Mario Giuliano ${ }^{9}$, Lucia Vassalli ${ }^{10}$, Patrizia Vici ${ }^{11}$, Giovanni Scognamiglio ${ }^{12}$, Daniele Generali ${ }^{13}$, Alberto Zambelli ${ }^{14}$, Marco Colleoni ${ }^{15}$, Corrado Tinterri ${ }^{16}$, Francesco Scanzi ${ }^{17}$, Leonardo Vigna ${ }^{18}$, Paola Scavina ${ }^{19}$, Teresa Gamucci ${ }^{20}$, Emilia Marrazzo ${ }^{21}$, Angelo Fedele Scinto ${ }^{22}$, Rossana Berardi ${ }^{23}$, Maria Agnese Fabbri ${ }^{24}$, Graziella Pinotti ${ }^{25}$, Daniela Franco ${ }^{26}$, Daniela Andreina Terribile ${ }^{2}$, Giuseppe Tonini ${ }^{27}$, Daniela Cianniello ${ }^{28}$ and Sandro Barni ${ }^{29}$

Clinicopathological prognostic features have limited value to identify with precision newly diagnosed patients with hormone receptor (HR)-positive, HER2-negative breast cancer (BC), who would benefit from chemotherapy (CT) in addition to adjuvant hormonal therapy (HT). The 21-gene Oncotype DX Breast Recurrence Score ${ }^{\oplus}$ (RS) assay has been demonstrated to predict CT benefit, hence supporting personalized decisions on adjuvant CT. The multicenter, prospective, observational study PONDx investigated the real-life use of $\mathrm{RS}^{\oplus}$ results in Italy and its impact on treatment decisions. Physicians' treatment recommendations $(\mathrm{HT} \pm \mathrm{CT})$ were documented before and after availability of RS results, and changes in recommendations were determined. In the HR + HER2 - early BC population studied $(N=1738)$, physicians recommended $C T+H T$ in $49 \%$ of patients pre-RS. RS-guided treatment decisions resulted in $36 \%$ reduction of $C T$ recommendations. PONDx confirms that RS results provide clinically relevant information for CT recommendation in early-stage BC, resulting in a reduction of more than a third of CT use.

npj Breast Cancer (2021)7:47; https://doi.org/10.1038/s41523-021-00246-4

\section{INTRODUCTION}

Breast cancer $(\mathrm{BC})$ is the most common type of malignancy in women and the second most common cancer overall. According to the GLOBOCAN database, the age-standardized incidence rate in Italy is 92.8 cases per 100,000 women per year ${ }^{1}$.

Classification of $\mathrm{BC}$ is primarily based on the expression of key signaling molecules including receptors for the female sex hormones (HR) estrogen and progesterone (ER, PR), as well as the human epidermal growth factor 2 (HER2).

For the large population of patients with $\mathrm{HR}+(\mathrm{ER}+$ and/or PR + ), HER2 - tumor status ( 70\% of nonmetastatic primary BCs) ${ }^{2}$, adjuvant hormonal therapy (HT) is recommended and is used as the standard treatment for most patients. When adjuvant chemotherapy (CT) is given in addition to HT, the side effects and risk burden require adequate measures to best identify the patients who will most likely derive a clinical benefit.

Traditionally, a set of clinical and pathological features have been used to evaluate the prognosis of the patient and guide decisions on adjuvant therapies. Besides the HR status, prognostic parameters include patient age, lymph node involvement, tumor size, histological type, and grade, as well as the Ki67 proliferation marker ${ }^{3}$. However, these conventional clinical and histopathological markers have insufficient specificity and sensitivity to precisely predict which patients are likely to experience a significant benefit of $C T$ on cancer recurrence that outweighs the substantial side effects.
Conventional parameters apparently have limited association with tumor biology, leaving a broad margin of predictive uncertainty ${ }^{4}$. Accordingly, only a minority of HR+, HER2- patients appears to benefit from CT: in a large meta-analysis performed by the Early Breast Cancer Trialists' Collaborative Group, the reduction of the 10-year recurrence rate by adjuvant CT was $<10 \%{ }^{5}$. Recently, the prospective ECOG-ACRIN Trial Assigning Individualized Options for Treatment (TAILORx) established in over 10,000 patients that the vast majority of $\mathrm{HR}+, \mathrm{HER} 2-$, node-negative (N0) primary BC patients (about to 80\%) do not derive benefit from CT in terms of recurrence risk ${ }^{6}$.

The 21-gene Oncotype DX Breast Recurrence Score multigene assay was developed to aid physicians in making personalized $C T$ treatment decisions in $\mathrm{HR}+$, HER2 - early-stage BC patients. Clinical validation and utility of the assay have been demonstrated in multiple studies with $>96,000 \mathrm{~N} 0$ and node-positive $(\mathrm{N}+) \mathrm{BC}$ patients worldwide $^{6-12}$.

Two studies have validated the prediction of a CT benefit by the Oncotype DX test for N0 patients with level $1 \mathrm{~B}$ and $1 \mathrm{~A}$ evidence. NSABP $B-20^{13}$ was a prospective analysis of archived, preserved samples and it demonstrated that patients with Recurrence Score (RS) results of 26-100 derive a substantial benefit from CT, whereas patients with an RS 0-10 had excellent clinical outcomes at 9 years with endocrine therapy alone ${ }^{14}$. The TAILORx study prospectively assessed in a large, randomized population the merits of CT in patients with RS 11-25 and demonstrated that, overall, they did

\footnotetext{
Università La Sapienza di Roma, Dipartimento Medicina Clinica e Molecolare, Rome, Italy. ${ }^{2}$ Policlinico Universitario Agostino Gemelli, IRCCS, Roma, Italy. ${ }^{3}$ IRCCS Regina Elena National Cancer Institute, Rome, Italy. ${ }^{4}$ Fondazione IRCCS Istituto Nazionale dei Tumori, Milano, Italy. ${ }^{5}$ Policlinico Sant'Orsola Malpighi, Bologna, Italy. ${ }^{6}$ Ospedale San Raffaele, Milano, Italy. ${ }^{7}$ Ospedale Gaetano Bernabeo, Ortona, Italy. ${ }^{8}$ Policlinico Umberto I, Roma, Italy. ${ }^{9}$ Azienda Ospedaliera Universitaria Federico II, Napoli, Italy. ${ }^{10}$ ASST Spedali Civili, Brescia, Italy. ${ }^{11}$ IRCCS Regina Elena National Cancer Institute, Roma, Italy. ${ }^{12}$ Ospedale Valduce, Como, Italy. ${ }^{13}$ ASST di Cremona, Cremona, Italy. ${ }^{14}$ ASST Papa Giovanni XXIII, Bergamo, Italy. ${ }^{15}$ Istituto Europeo di Oncologia, Milano, Italy. ${ }^{16}$ Istituto Clinico Humanitas, Rozzano, Italy. ${ }^{17}$ IRCCS Multimedica, Sesto San Giovanni, Italy. ${ }^{18}$ Azienda Ospedaliera San Camillo Forlanini, Roma, Italy. ${ }^{19}$ Azienda Ospedaliera San Giovanni - Addolorata, Roma, Italy. ${ }^{20}$ Ospedale SS. Trinità, Sora, Italy. ${ }^{21}$ Istituto Clinico Humanitas, Rozzano, Italy. ${ }^{22}$ Ospedale San Giovani Calibita Fatebenefratelli, Roma, Italy. ${ }^{23}$ Azienda Ospedaliero Universitaria Ospedali Riuniti di Ancona, Torrette, Italy. ${ }^{24}$ Ospedale di Belcolle, Viterbo, Italy. ${ }^{25}$ Ospedale di Circolo e Fondazione Macchi, Varese, Italy. ${ }^{26}$ Ospedale Nuovo Regina Margherita, Roma, Italy. ${ }^{27}$ Policlinico Universitario Campus Biomedico, Roma, Italy. ${ }^{28}$ Istituto Nazionale Tumori Fondazione G. Pascale, Napoli, Italy. ${ }^{29}$ ASST BG Ovest Ospedale Treviglio, Treviglio, BG, Italy. ${ }^{凶}$ email: francesco.cognetti@ifo.gov.it
} 
not derive a significant benefit from CT. Taken together, these results established that the Oncotype DX assay can guide CT treatment decisions such that N0 patients with RS 0-25 can safely forego $C T$, whereas patients with RS 26-100 do derive substantial benefit from CT used in addition to hormonal treatment. In postmenopausal women with $\mathrm{N}+, \mathrm{ER}+\mathrm{BC}$, the prospective, retrospective analysis of SWOG-8814 trial established that N1 patients with RS $0-17$ could be safely spared $C T$, whereas patients with RS 31-100 achieved a strong clinical benefit with $C T^{15}$.

For NO and N+ disease, aggregate data from prospective registries with 5-10 years of observation confirm that the RS result consistently identifies patients with good clinical outcomes when treated with HT alone $e^{10,16,17}$.

The Oncotype DX assay has been incorporated into clinical and pathological guidelines of major international medical societies including the European Society of Medical Oncology, St. Gallen Consensus Conference, American Society of Clinical Oncology, and American Joint Committee on Cancer. The National Comprehensive Cancer Network Guidelines state that the Oncotype DX assay is the only test with proven validity to predict CT benefit ${ }^{18}$.

In decision impact studies in Europe conducted before publication of the TAILORx study and, hence, before the predictive cutoffs were established with precision, outcomes have shown the utility of the RS result in clinical practice. In a meta-analysis of more than $500 \mathrm{~N} 0, \mathrm{HR}+$, HER2 - primary BC patients from four studies, the overall rate of recommendation change was $32 \%$ post- vs. pre-testing, whereas the $\mathrm{CT}$ recommendation rate decreased from $55 \%$ to $34 \%{ }^{19}$.

Here we describe the results of the multicenter, prospective, observational study, PONDx, which was performed in Italy from February 2016 to December 2017, and which investigated the real-life use of the Oncotype DX Breast Recurrence Score test by physicians treating early $B C$ patients in routine care in clinical $B C$ reference centers. The study primarily evaluated the impact of the Oncotype DX assay on physicians' treatment decisions. A further objective was the characterization of the patient population in which the test is used in real-life settings at clinical BC reference centers in Italy.

\section{RESULTS}

Data from 1738 BC patients who underwent Oncotype DX testing were available from 27 reference centers located in 6 regions of Italy (Lombardia, Lazio, Emilia Romagna, Campania, Abruzzo, and Marche). In the present analysis, 14 patients were excluded due to incomplete data, leaving 1724 in the analysis cohort.

The tumors diagnosed in the participating patients were mostly invasive ductal HR-positive carcinomas, with histological grade 2 and 3 , and tumor size ranging from 1 to $5 \mathrm{~cm}$, with Ki67 expression mostly in the range from $10 \%$ to $>30 \%$. The majority of patients were $>50$ years old; $36 \%$ were premenopausal and $55 \%$ postmenopausal (Table 1).

The distribution of Recurrence Score results is shown in Table 2 (left columns). Judged by the conventional cut points, $57 \%$ of the overall population were in the RS $0-17,34 \%$ in the RS $18-30$ and 9\% in the RS $31-100$ group. Using the TAILORx-based cut points, $83 \%$ of the population was in the RS $0-25$ group and $17 \%$ in the RS 26-100 group.

It is interesting to note the discordance of some of the key classical pathological parameters and RS results. Using the TAILORx-based RS cut points (Table 2, right columns), the discordance between some classical pathological parameters and RS result remains pronounced, with a significant proportion of grade 2 (89\%) and grade $3(64 \%)$ patients having RS 0-25, indicating no CT benefit. Similarly, patients with the lowest $(<10 \%)$ or highest $(>30 \%)$ level of expression of the proliferation marker Ki67 were found to have RS $26-100$ and RS 0-25, respectively. The latter may in part be related to the known lack of reproducibility of

\begin{tabular}{|c|c|c|c|}
\hline Parameter & Characteristics & $\begin{array}{l}\text { Number of } \\
\text { patients }\end{array}$ & $\begin{array}{l}\text { Percentage of } \\
\text { patients }\end{array}$ \\
\hline \multirow[t]{4}{*}{ Age } & $<35$ Years & 26 & $1 \%$ \\
\hline & $35-50$ Years & 655 & $38 \%$ \\
\hline & 51-70 Years & 831 & $48 \%$ \\
\hline & $>70$ Years & 226 & $13 \%$ \\
\hline \multirow[t]{2}{*}{ Gender } & Female & 1720 & $99 \%$ \\
\hline & Male & 18 & $1 \%$ \\
\hline \multirow[t]{4}{*}{ Menopausal status } & Pre & 623 & $36 \%$ \\
\hline & Peri & 137 & $8 \%$ \\
\hline & Post & 960 & $55 \%$ \\
\hline & ND & 18 & $1 \%$ \\
\hline \multirow{3}{*}{ Histological subtype } & Other & 110 & $6 \%$ \\
\hline & Ductal & 1417 & $82 \%$ \\
\hline & Lobular & 211 & $12 \%$ \\
\hline \multirow[t]{3}{*}{ Histological grade } & G1 & 165 & $9 \%$ \\
\hline & $\mathrm{G} 2$ & 1090 & $63 \%$ \\
\hline & G3 & 483 & $28 \%$ \\
\hline \multirow[t]{4}{*}{ Tumor size } & $<1 \mathrm{~cm}$ & 232 & $13 \%$ \\
\hline & $1-2 \mathrm{~cm}$ & 1052 & $61 \%$ \\
\hline & $2.1-5 \mathrm{~cm}$ & 432 & $25 \%$ \\
\hline & $>5 \mathrm{~cm}$ & 22 & $1 \%$ \\
\hline \multirow[t]{3}{*}{ Nodal status } & No & 1192 & $69 \%$ \\
\hline & Nmic & 113 & $7 \%$ \\
\hline & N1 & 433 & $25 \%$ \\
\hline \multirow[t]{2}{*}{ ER status } & Negative & 5 & $<1 \%$ \\
\hline & Positive & 1733 & $>99 \%$ \\
\hline \multirow[t]{2}{*}{ PR status } & Negative & 164 & $9 \%$ \\
\hline & Positive & 1574 & $91 \%$ \\
\hline \multirow[t]{3}{*}{ HER2 status } & Equivocal & 72 & $4 \%$ \\
\hline & Negative & 1637 & $94 \%$ \\
\hline & Positive & 29 & $2 \%$ \\
\hline \multirow[t]{5}{*}{ Ki67 expression } & $<10 \%$ & 169 & $10 \%$ \\
\hline & $10-20 \%$ & 576 & $33 \%$ \\
\hline & $21-30 \%$ & 611 & $35 \%$ \\
\hline & $>30 \%$ & 377 & $22 \%$ \\
\hline & ND & 5 & $<1 \%$ \\
\hline
\end{tabular}

Ki67 assays ${ }^{20-22}$, yet could be expected considering Ki67 has not been demonstrated to correlate with $C T$ response ${ }^{23}$. No discernible correlations were found between RS result and tumor size, age, or menopausal status.

In the population analyzed for treatment recommendation $(n=$ 1683), the physicians recommended CT $+\mathrm{HT}$ in 824 patients $(49 \%)$ prior to the availability of the RS result, whereas 859 (51\%) were assigned to HT alone. Patients with recommendations for other therapies than $\mathrm{CT}$ or $\mathrm{CT}+\mathrm{HT}$ were excluded from this analysis.

After the RS results became available, the physicians changed their decision in 512 patients (30\%). Consequently, the number of patients with a $\mathrm{CT}+\mathrm{HT}$ recommendation dropped from 824 to 524 , corresponding to a net reduction of CT recommendations of 36\% (Table 3 and Fig. 1).

Looking at selected groups defined by tumor characteristics, the following picture emerges among the patients with 
TABLE 2. Oncotype DX Breast Recurrence Score ${ }^{\circledR}$ results categorized according to RS groups used prior to TAILORx (left) and RS groups based on TAILORx cut points (shaded columns, right).

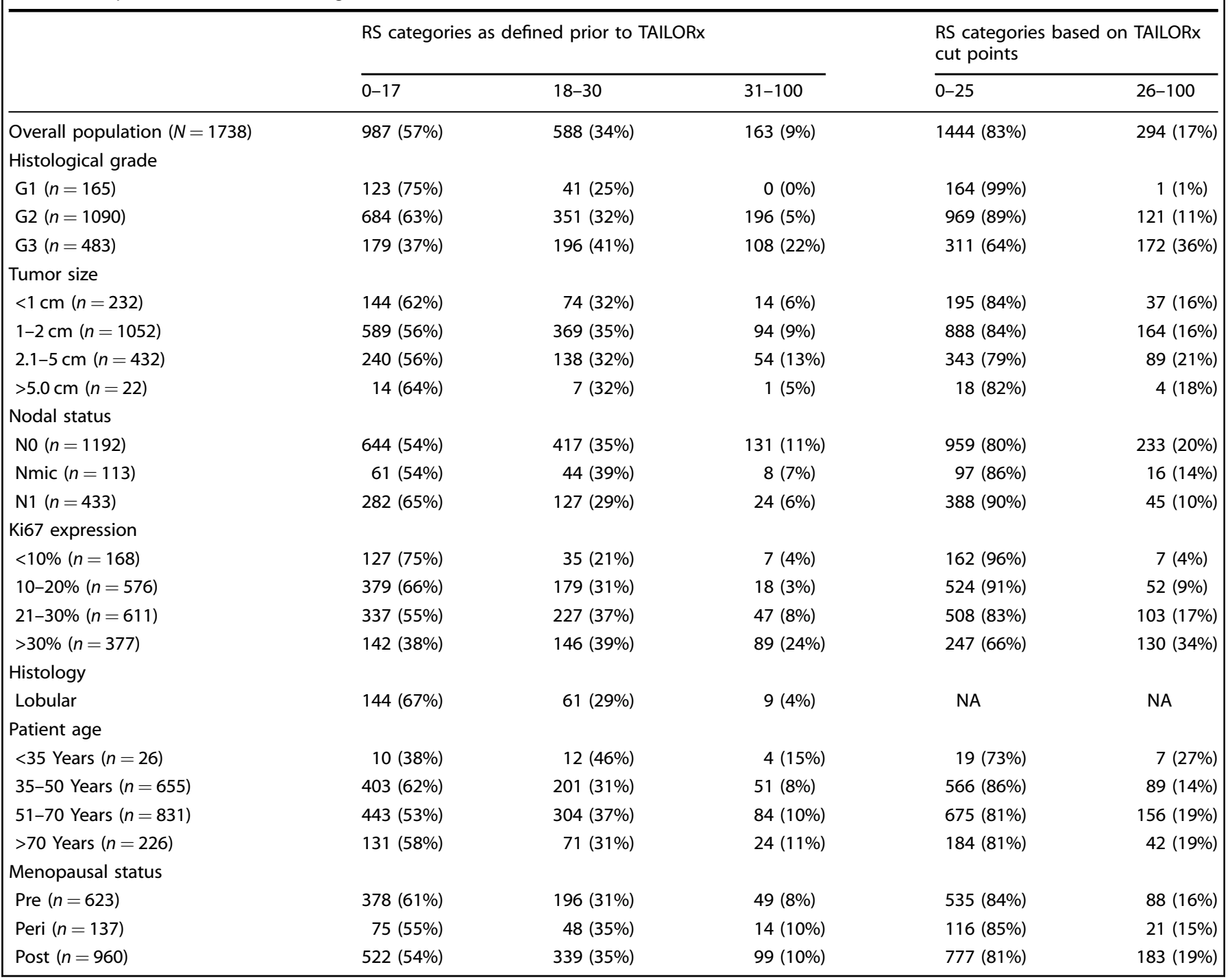

TABLE 3. Recommendations by the treating physician regarding adjuvant anti-tumor therapy before (PRE-RS) and after availability (POST-RS) of the Recurrence Score result. For these analyses, patients with recommendations other than $\mathrm{CT}+\mathrm{HT}$ or HT were excluded.

\begin{tabular}{|c|c|c|c|c|c|c|}
\hline \multirow[b]{2}{*}{ Population } & & \multicolumn{4}{|c|}{ Treatment recommendations, $n$ (\%) } & \multirow{2}{*}{$\begin{array}{l}\text { Change in } \mathrm{CT}+\mathrm{HT} \\
\text { recommendations (\%) }\end{array}$} \\
\hline & & $\mathrm{CT}+\mathrm{HT}$ & HT & $\mathrm{CT}+\mathrm{HT}$ & HT & \\
\hline Overall population & $N=1683$ & $824(49 \%)$ & $859(51 \%)$ & $524(31 \%)$ & 1159 (69\%) & $-36 \%$ \\
\hline No & $n=1160$ & $512(44 \%)$ & $648(56 \%)$ & $374(32 \%)$ & $786(68 \%)$ & $-27 \%$ \\
\hline Nmic & $n=109$ & $54(50 \%)$ & 55 (50\%) & $33(30 \%)$ & 76 (70\%) & $-39 \%$ \\
\hline Grade 3 & $n=475$ & $350(74 \%)$ & $125(26 \%)$ & $254(53 \%)$ & $221(47 \%)$ & $-27 \%$ \\
\hline Ki67 $>20 \%$ & $n=962$ & $608(63 \%)$ & $354(37 \%)$ & $386(40 \%)$ & $576(60 \%)$ & $-37 \%$ \\
\hline Lobular breast cancer & $n=203$ & $98(52 \%)$ & $10548 \%)$ & $46(23 \%)$ & $157(77 \%)$ & $-53 \%$ \\
\hline Age $>50$ years & $n=1027$ & $464(45 \%)$ & $563(55 \%)$ & $314(31 \%)$ & $713(69 \%)$ & $-31 \%$ \\
\hline Age $\leq 50$ years & $n=656$ & $360(55 \%)$ & 296 (45\%) & $210(32 \%)$ & $446(68 \%)$ & $-42 \%$ \\
\hline
\end{tabular}




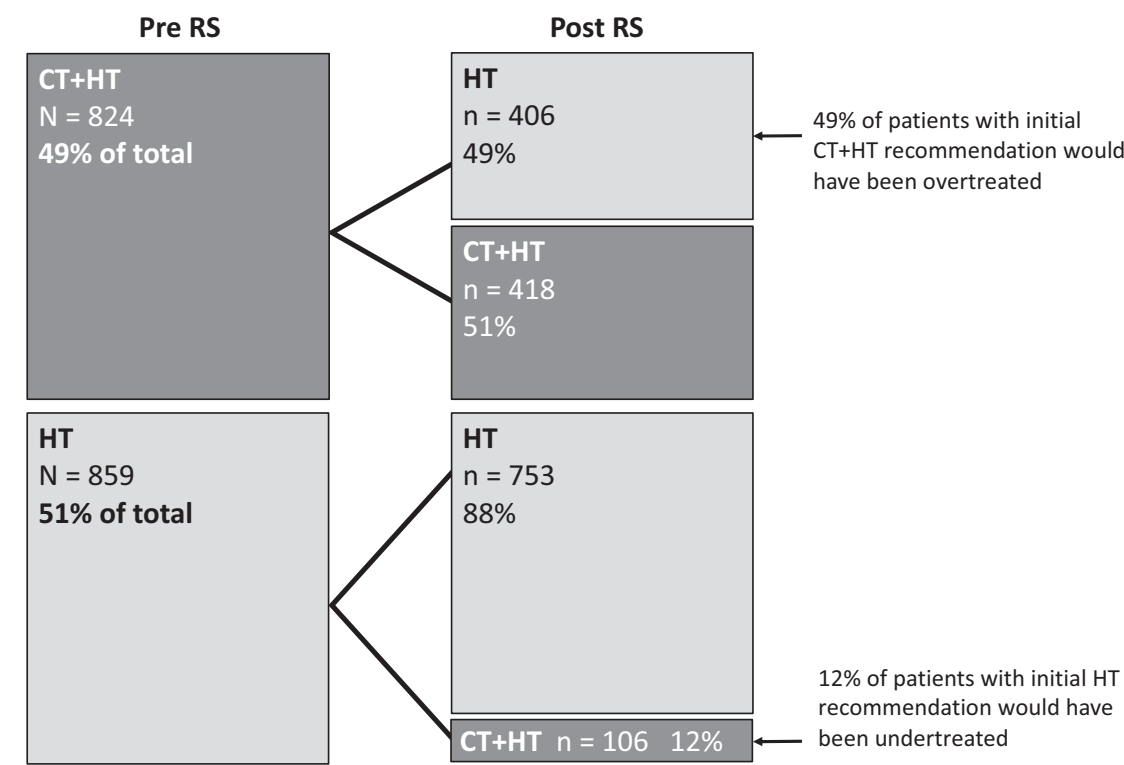

Fig. 1 Changes in treatment recommendations before and after availability of the Recurrence Score result. Rates of hormone therapy alone $(\mathrm{HT})$ or chemo-endocrine therapy $(\mathrm{CT}+\mathrm{HT})$ recommendations before testing (Pre RS) and changes in recommendations based on the test resutls (Post RS).

\begin{tabular}{|c|c|c|c|c|c|}
\hline Nodal status & Patient age & RS 0-15 & RS 16-20 & RS $21-25$ & RS 26-100 \\
\hline \multirow[t]{3}{*}{ No } & $\leq 50$ Years & HT-only & HT-only 90\% & HT-only 60\% & $\mathrm{CT}+\mathrm{HT}$ \\
\hline & & & $\mathrm{CT}+\mathrm{HT} 10 \%$ & $\mathrm{CT}+\mathrm{HT} 40 \%$ & \\
\hline & $>50$ Years & HT-only & & & $\mathrm{CT}+\mathrm{HT}$ \\
\hline
\end{tabular}

clinicopathological high-risk tumors (Table 3 ): in patients with grade 3 malignancies $(n=475)$, physicians changed their treatment recommendation in $37 \%$ of cases, leading to a $27 \%$ net reduction of $\mathrm{CT}+\mathrm{HT}$. Patients with Ki67 expression $>20 \%$ $(n=962)$ had their recommendation amended in $36 \%$, with a $37 \%$ net reduction in $\mathrm{CT}+\mathrm{HT}$ recommendations (from 608 to 386 patients).

For patients with N0 nodal status $(n=1160)$ CT + HT recommendations declined by $27 \%$ (from 512 to 374 patients). In contrast, the group with N1 disease $(n=414)$ saw the frequency of $\mathrm{CT}+\mathrm{HT}$ recommendations reduced by a net percentage of $55 \%$ after the RS results became available (from 258 to 110 patients). Similarly, pronounced effects were observed for the subset of patients with Nmic $(n=109)$ and with lobular BC $(n=206)$. In these specific groups, the net reduction of CT $+\mathrm{HT}$ was $39 \%$ and $53 \%$, respectively.

Regarding patients aged $>50$ vs. $\leq 50$ years, both age groups showed a strong net reduction of $\mathrm{CT}+\mathrm{HT}$ recommendations (by $31 \%$ and $42 \%$, respectively). In the group with initial recommendations of HT-only $(N=859)$, a minor fraction of patients had their recommended treatment changed to $\mathrm{CT}+\mathrm{HT}(n=102 ; 12 \%)$.

A simulation of expected treatment recommendations after availability of the RS result was performed based on TAILORX RS cut points ${ }^{6}$ and the estimated interpretation of the RS results according to the findings of the TAILORx study (see "Methods" and Table 4). According to these premises, 75\% $(n=1263)$ of patients would receive HT-only and $25 \%(n=420)$ adjuvant CT + $\mathrm{HT}$ regimens in this setting, corresponding to a relative reduction of $49 \%$ for the overall population (Fig. 2) (47\% for N0) (Table 5). Regarding the age groups $>50$ vs. $\leq 50$ years, there was a net reduction of $\mathrm{CT}+\mathrm{HT}$ recommendations (by $41 \%$ and $50 \%$, respectively). The proportion of patients $\leq 50$ years with NO disease and RS 16-20 and RS 21-25 represent, respectively, 9\% and $5 \%$ of the overall NO population.

\section{DISCUSSION}

In this observational study, we documented the use of the Oncotype DX assay and its impact on physicians' therapeutic recommendations in a sizeable patient population from $27 \mathrm{BC}$ reference centers in 6 regions of Italy. Our data add to the growing body of evidence from RS result decision impact studies with NO and $\mathrm{N}+$ tumors performed in Europe ${ }^{19,24-26}$, North America ${ }^{27,28}$, or Australia ${ }^{29}$.

Assessment of the recurrence risk by the Oncotype DX Breast Recurrence Score assay influenced physician's choice of adjuvant regimens, resulting in an overall reduction of $36 \%$ in CT recommendations vs. decisions based on prognostic-only clinicopathological risk parameters. Recalculation of the expected postRS recommendations using estimates based on TAILORx cut points and results showed a higher reduction (49\%) of the proportion of patients recommended CT in the total population. This observation was consistent in both age groups of $\leq 50$ and $>50$ years.

The significant overall reduction in CT recommendations guided by RS results in this study correlates with that of other studies published worldwide, reporting up to $47 \%$ reduction with similar patient populations and pre-TAILORx cut points. This indicates a consistent decision impact of the RS results ${ }^{19,24-29}$. The relative reduction in CT recommendations is relevantly influenced by 


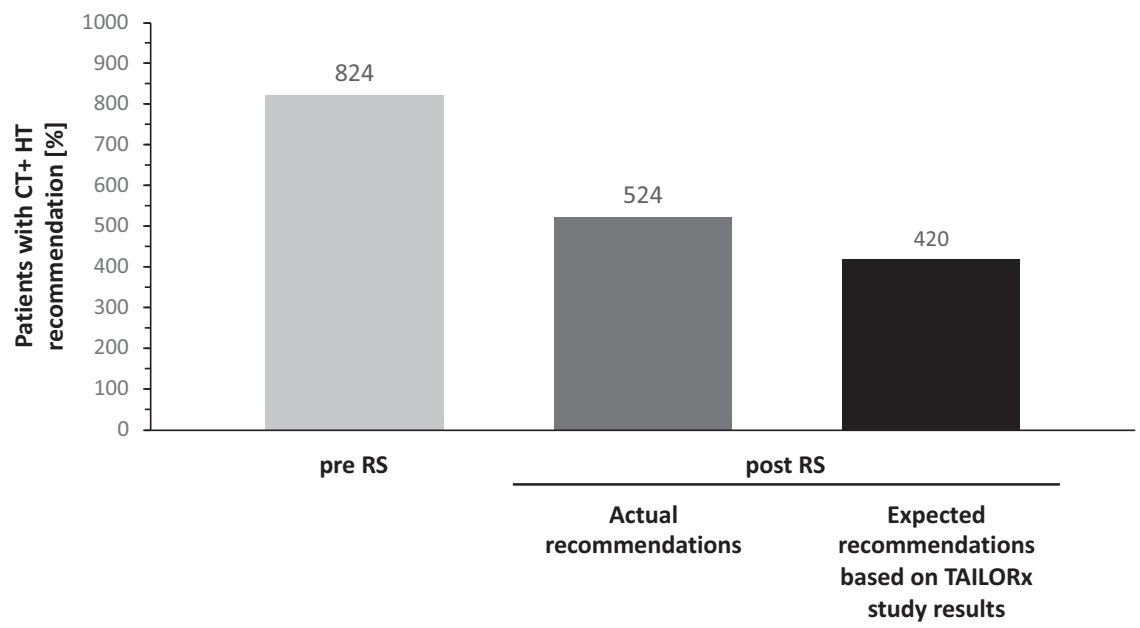

Fig. 2 Patients with post-RS recommendations for chemo-endocriine therapy (CT + HT): actual number based on previous RS cut points and expected percentage assuming decision-making according to TAILORx results $(\mathbf{N}=\mathbf{1 6 8 3})$. Pre RS: treatment recommendations before avalability of the Recurrence Score result. Post RS: treatment recommendations accounting for the Recurrence Score result.

\begin{tabular}{|c|c|c|c|c|c|}
\hline & \multicolumn{2}{|l|}{ PRE-RS } & \multicolumn{2}{|l|}{ POST-RS } & \multirow{2}{*}{$\begin{array}{l}\text { Change in } \mathrm{CT}+\mathrm{HT} \\
\text { recommendations (\%) }\end{array}$} \\
\hline & $\mathrm{CT}+\mathrm{HT}$ & HT & $\mathrm{CT}+\mathrm{HT}$ & HT & \\
\hline$N=1683$ & 824 (49\%) & 859 (51\%) & $420(25 \%)$ & $1263(75 \%)$ & $-49 \%$ \\
\hline$N=1160$ & $512(44 \%)$ & 648 (56\%) & $270(23 \%)$ & 890 (77\%) & $-47 \%$ \\
\hline
\end{tabular}

clinical practice and baseline CT usage. This has been reported to be highly variable across countries, centers, and within single centers ${ }^{4}$

The findings of our study support previous evidence ${ }^{4}$ demonstrating physicians' uncertainty to recommend adjuvant treatment relying on prognostic-only factors that have not been correlated with the prediction of CT benefit. Tumor grade and size, in particular, are not stringently related to tumor biology. Although they estimate patient prognosis, i.e., risk of recurrence, they do not predict response to CT as a specific treatment option ${ }^{23}$.

In addition, the high variability and lack of standardization on Ki67 or histological tumor-grade assessments leave the treating physicians with a margin of uncertainty ${ }^{22}$. The Oncotype DX test results can provide confidence for $\mathrm{CT}$ treatment decisions with a strong body of evidence on the prediction of CT benefit ${ }^{1}$. Of note, no patient subgroup in this study was identified, which could forego Oncotype DX RS testing without losing potentially useful predictive information on $\mathrm{CT}$ benefit or lack thereof.

Analysis of the widely used Ki67 proliferation marker revealed that $66 \%$ of the patients with high Ki67 expression (>30\%) had an RS result 0-25 and would not be expected to derive benefit from $\mathrm{CT}$, indicating that Ki67 should probably not be used as a dominant indicator for treatment decisions. Similar considerations apply for patients with grade 3 tumors, $64 \%$ of whom had an RS result in the $0-25$ range. This is consistent with observations in the TAILORx study where $73 \%$ of patients with a high clinical risk based on tumor-grade and size assessment had an RS result 0-25 and hence might have been overtreated if the RS result had not been used for the treatment decision ${ }^{6}$.

Conversely, a minor fraction of patients had their initial HT-only recommendations changed to actually receive additional $\mathrm{CT}$, suggesting that they were rescued from potential undertreatment based on conventional criteria.
The overall change in CT recommendations by Oncotype DX RS results in this study, based on pre-TAILORx cut points, is in the range of that in other published studies with similar patient populations worldwide, indicating a consistent interpretation of the RS results ${ }^{19,24-29}$. The strength of the present study lies in its size: this is the largest decision impact study reported to date. Furthermore, this real-life study confirms the results of a randomized controlled trial. Its main limitation is that the study was performed before the availability of TAILORx data, demonstrating that even a higher proportion of patients could be spared $\mathrm{CT}$, thanks to the RS results. Consequently, we might underestimate the net CT sparing effect of the Oncotype DX test. Although the results of the TAlLORx study were practice-changing for NO patients, the ongoing RxPONDER trial will provide additional information on the clinical usefulness of the Oncotype DX assay in women with HR-positive BC and positive axillary nodes. In this study, we observe a very significant reduction of CT recommendations (55\%) for patients with limited nodal involvement (N1). This is supported by the consistent evidence from the SWOG-8814 ${ }^{15}$ and Plan $B^{8}$ studies, as well as prospective registries $^{9,11}$ supporting $C T$ sparing for patients with the lowest $\mathrm{RS}$ results.

Chemotherapeutic regimens used in the adjuvant setting in women with early $\mathrm{BC}$ are associated with a significant risk of acute and long-term adverse effects - the latter including fatigue, cardiotoxicity, cognitive impairment ${ }^{30}$, peripheral neuropathy, and cases of secondary malignancies including leukemia. Quality of life and working ability may be reduced at least temporarily by these treatments as well. Therefore, tools refining the population that derives appropriate benefit to justify the adverse effects of adjuvant CT serve an important medical need in patients with HR + , HER2 - primary BC. The Oncotype DX assay is such an instrument and may contribute significantly to a reduction in the use of $\mathrm{CT}$ for patients who are unlikely to derive benefit. 
Conversely, the assay allows identification of a group of $15-20 \%$ of $\mathrm{HR}+$, HER2 - early BC patients who derive a substantial benefit from $\mathrm{CT}$. In these patients, $\mathrm{CT}$ in addition to $\mathrm{HT}$ consistently results in lower distant recurrence rates than HT alone $\mathrm{H}^{5,13,14}$.

In our study, we reported, overall, $83 \%$ of patients had RS $0-25$ guiding towards a significant de-escalation of CT. These proportions are consistent with the TAILORx study and with patient registries such as the Surveillance, Epidemiology and End Results registry in the United States ${ }^{31}$ and the Clalit registry in Israel ${ }^{10}$, reporting $84 \%$ and $80 \%$ patients with RS $0-25$, respectively. This majority of patients (about $80 \%$ ) with RS 0-25 consistently showed excellent clinical outcome with HT alone and hence can safely be spared CT.

Results from the TAILORx study are considered practicechanging, because for the first time it was shown in a large prospective randomized trial that a sizeable group of patients could be identified with a unique multigene assay to derive minimal or no benefit from CT. For patients initially recommended to receive chemo-HT in our study, use of the Oncotype DX test with TAILORx cut points led to a reduction by nearly $50 \%$ in CT recommendations.

In addition to the clinical benefit to the patients who are spared adverse effects, reduction of CT use has relevant implications for the healthcare system through reduction of direct expenses (cytotoxic drugs and their application) and indirect costs (managing side effects). Benefits to healthcare-associated and societal costs importantly include diminished duration of absence from work, which has been shown to be significantly prolonged by a median of 7 months for patients who receive CT. In fact, the use of $\mathrm{CT}$ was one of the factors with the highest risk ratio of delayed time to work after primary $\mathrm{BC}^{32}$, responsible for more than a quarter of the total costs of $\mathrm{CT}^{33}$. Other genomic assays are available for early BC patients; however, it is noteworthy that although Oncotype DX brings value guiding $\mathrm{CT}$ decisions based on direct evidence of prediction of CT benefit, other genomic assays are prognostic-only. Decision impact studies with MammaPrint prognostic assay ${ }^{34,35}$, EndoPredict ${ }^{\oplus 36}$, or Prosigna ${ }^{\oplus 37,38}$ consistently reported a limited impact of net CT use related to a balance between significant reduction from CT-HT to CT and a significant increase from HT alone to CT-HT.

The observations on the use and impact of the Oncotype DX Breast Recurrence Score test on the participating reference centers of PONDx in Italy support the notion that the test provides clinically useful predictive information, complementing standard clinical and pathological risk parameters for patients with HR+, HER2 - N0/N1 primary BC. The physicians used the results to modify their original treatment recommendations, which resulted in a reduction of patients recommended for CT by more than a third.

Estimating the effects based on the RS categories and outcomes established in the recently completed large prospective TAILORx trial confirmed the primary results of PONDx, indicating the potential for an even more pronounced reduction of $C T$ recommendations and thereby potentially sparing a significant proportion of patients from acute and long-term toxicities of these treatments.

\section{METHODS}

\section{Patients eligibility}

Eligible patients fulfilled the validated criteria for use of the Oncotype DX assay: patients aged $\geq 18$ years with a recent diagnosis of early, singleinvasive ER+ HER2- BC and available information on lymph node involvement categorized as NO, Nmic (micrometastatic node involvement), or $\mathrm{N} 1$ (one to three positive nodes).

Baseline patient documentation included age and sex, menopausal status, conventional clinical and pathological tumor characteristics including histologic type (lobular/ductal), tumor size and grade, nodal status, receptor status (ER, PR, HER2), Ki67 expression, and RS results as soon as available.

\section{Ethics}

Patients provided written informed consent before participation in the study. The protocol was approved by the Ethics Committee of all participating institutions: Università La Sapienza di Roma, Policlinico Universitario Agostino Gemelli, IRCCS Regina Elena National Cancer Institute, Fondazione IRCCS Istituto Nazionale dei Tumori, Policlinico Sant'Orsola Malpighi, Ospedale San Raffaele, Ospedale Gaetano Bernabeo, Policlinico Umberto I, Azienda Ospedaliera Universitaria Federico II, ASST Spedali Civili, IRCCS Regina Elena National Cancer Institute, Ospedale Valduce, ASST di Cremona, ASST Papa Giovanni XXIII, Istituto Europeo di Oncologia, Istituto Clinico Humanitas, IRCCS Multimedica Sesto San Giovanni, Azienda Ospedaliera San Camillo Forlanini, Azienda Ospedaliera San Giovanni - Addolorata, Ospedale SS. Trinità, Istituto Clinico Humanitas, Ospedale San Giovani Calibita Fatebenefratelli, Azienda Ospedaliero Universitaria Ospedali Riuniti di Ancona, Ospedale di Belcolle, Ospedale di Circolo e Fondazione Macchi, Ospedale Nuovo Regina Margherita, Policlinico Universitario Campus Biomedico, Istituto Nazionale Tumori Fondazione G. Pascale, and ASST BG Ovest Ospedale Treviglio.

\section{Treatment decisions}

Prior to Oncotype DX testing, CT was recommended to patients with worse prognosis based on clinical, pathological, and biological features as per the local clinical practice. Individual treatment modalities (HT, CT-HT) recommended by the treating physician were documented before (preRS) and after (post-RS) availability of the test results. Patients followed recommendations that emerged post-RS result.

Descriptive analyses were performed for the overall population and subpopulations of patients with clinical high-risk tumors defined by grade 3 disease and/or $>20 \%$ Ki67 positivity, patients with N0 vs. N1 nodal status, and those with cancers of lobular histology. For these populations, changes in treatment recommendations regarding $\mathrm{HT}$ and/or $\mathrm{CT}$ were determined by comparing the percentage of patients receiving a recommendation of $\mathrm{HT}$ or $\mathrm{HT}+\mathrm{CT}$ before vs. after the test results became available to the treating physician. The primary analysis used the RS cut points commonly used before TAILORx was published, to define three RS groups: $0-17,18-30$, and 31-100.

An additional exploratory analysis regarding the influence of the RS result on treatment recommendations was performed using the RS cut points for NO patients defined by the TAILORx trial and their expected interpretation in clinical practice. In the TAILORx study, exploratory analyses suggested that all NO patients above the age of 50 years with RS 0-25 have no CT benefit. For younger patients ( $\leq 50$ years), an RS of 0-15 indicated no CT benefit, whereas some CT benefit was derived for RS 16-20 (1.6\%) and RS 21-25 (6.5\%). Patients with N1 disease were assigned to RS groups according to previous cut points as described above. Thus, the algorithm described in Table 4 was recommended by an expert panel based on the finding from exploratory analyses of TAILORx, suggesting a potentially clinically meaningful benefit from $C T$ for a small number of patients, and was used for patients with nodal status NO in the analysis of presumed treatment recommendations based on TAILORx findings.

\section{Reporting summary}

Further information on research design is available in the Nature Research Reporting Summary linked to this article.

\section{DATA AVAILABILITY}

The datasets that support the findings of this study will be made available upon reasonable request from the corresponding author, Dr. Francesco Cognetti, email address: francesco.cognetti@ifo.gov.it. The data generated and analyzed during this study are described in the following metadata record ${ }^{39}$ : https://doi.org/10.6084/m9. figshare.13049816.

Received: 11 November 2019; Accepted: 12 November 2020; Published online: 05 May 2021

\section{REFERENCES}

1. Ferlay, J. et al. Estimating the global cancer incidence and mortality in 2018: GLOBOCAN sources and methods. Int. J. Cancer 144, 1941-1953 (2019).

2. Howlader, N. et al. US incidence of breast cancer subtypes defined by joint hormone receptor and HER2 status. J. Natl Cancer Inst. 106, 5 (2014). 
3. Curigliano, G. et al. De-escalating and escalating treatments for early-stage breast cancer: the St. Gallen International Expert Consensus Conference on the Primary Therapy of Early Breast Cancer 2017. Ann. Oncol. 28, 1700-1712 (2017).

4. Aapro, M. et al. The MAGIC survey in hormone receptor positive $(\mathrm{HR}+)$, HER2negative (HER2-) breast cancer: When might multigene assays be of value? Breast 33, 191-199 (2017).

5. Early Breast Cancer Trialists' Collaborative Group (EBCTCG) et al.Comparisons between different polychemotherapy regimens for early breast cancer: metaanalyses of long-term outcome among 100,000 women in 123 randomised trials. Lancet 379, 432-444 (2012).

6. Sparano, J. A. et al. Adjuvant chemotherapy guided by a 21-gene expression assay in breast cancer. N. Engl. J. Med. 379, 111-121 (2018).

7. Sparano, J. A. et al. Prospective validation of a 21 -gene expression assay in breast cancer. N. Engl. J. Med. 373, 2005-2014 (2015)

8. Nitz, U. et al. Reducing chemotherapy use in clinically high-risk, genomically lowrisk pN0 and $\mathrm{pN} 1$ early breast cancer patients: five-year data from the prospective, randomised phase 3 West German Study Group (WSG) PlanB trial. Breast Cancer Res. Treat. 165, 573-583 (2017).

9. Stemmer, S. M. et al. Clinical outcomes in ER+ HER2-node-positive breast cancer patients who were treated according to the Recurrence Score results: evidence from a large prospectively designed registry. NPJ Breast Cancer 3, 32 (2017).

10. Stemmer, S. M. et al. Clinical outcomes in patients with node-negative breast cancer treated based on the recurrence score results: evidence from a large prospectively designed registry. NPJ Breast Cancer 3, 33 (2017).

11. Roberts, M. C. et al. Breast cancer-specific survival in patients with lymph nodepositive hormone receptor-positive invasive breast cancer and Oncotype DX Recurrence Score results in the SEER database. Breast Cancer Res. Treat. 163, 303-310 (2017)

12. Shak, S. et al. Outcome disparities by age and 21-gene recurrence score ${ }^{\circledR}$ (RS) result in hormone receptor positive (HR+) breast cancer (BC). Ann. Oncol. 27, VI43 (2016)

13. Paik, S. et al. Gene expression and benefit of chemotherapy in women with nodenegative, estrogen receptor-positive breast cancer. J. Clin. Oncol. 24, 3726-3734 (2006).

14. Geyer, C. E. Jr et al. 21-Gene assay as predictor of chemotherapy benefit in HER2negative breast cancer. NPJ Breast Cancer 4, 37 (2018).

15. Albain, K. S. et al. Prognostic and predictive value of the 21-gene recurrence score assay in postmenopausal women with node-positive, oestrogen-receptorpositive breast cancer on chemotherapy: a retrospective analysis of a randomised trial. Lancet 11, 55-65 (2010).

16. Mamounas, E. P. et al. Clinical relevance of the 21-gene Recurrence Score ${ }^{\circledR}$ assay in treatment decisions for patients with node-positive breast cancer in the genomic era. NPJ Breast Cancer 4, 27 (2018).

17. Hortobagyi, G.N. et al. Breast cancer-specific mortality (BCSM) in patients (pts) with node-negative (NO) and node-positive $(\mathrm{N}+)$ breast cancer $(\mathrm{BC})$ guided by the 21-gene assay: a SEER-genomic population-based study. Cancer Res. 79, P311-02 (2019)

18. National Comprehensive Cancer Network. NCCN Clinical Practice Guidelines in Oncology; Breast Cancer Version 3.2018, www.NCCN.org (2018).

19. Albanell, J. et al. Pooled analysis of prospective European studies assessing the impact of using the 21-gene Recurrence Score assay on clinical decision making in women with oestrogen receptor-positive, human epidermal growth factor receptor 2-negative early-stage breast cancer. Eur. J. Cancer 66, 104-113 (2016).

20. Polley, M. Y. et al. An international Ki67 reproducibility study. J. Natl Cancer Inst 105, 1897-1906 (2013).

21. Varga, Z. et al. How reliable is Ki-67 immunohistochemistry in grade 2 breast carcinomas? A QA study of the Swiss Working Group of Breast- and Gynecopathologists. PLOS ONE 7, e37379 (2012).

22. Dowsett, M. et al. Assessment of Ki67 in breast cancer: recommendations from the International Ki67 in Breast Cancer working group. J. Natl Cancer Inst. 103, 1656-1664 (2011).

23. Viale, G. et al. Predictive value of tumor Ki-67 expression in two randomized trials of adjuvant chemoendocrine therapy for node-negative breast cancer. J. Nat Cancer Inst. 100, 207-212 (2008).

24. Curtit, E. et al. Results of PONDx, a prospective multicenter study of the oncotype DX ${ }^{\circledR}$ breast cancer assay: real-life utilization and decision impact in French clinical practice. Breast 44, 39-45 (2019).

25. Dieci, M. V. et al. First prospective multicenter Italian study on the impact of the 21-gene Recurrence Score in adjuvant clinical decisions for patients with ER positive/HER2 negative breast cancer. Oncologist 23, 297-305 (2018).

26. Pestalozzi, B. C. et al. Adjuvant treatment recommendations for patients with ERpositive/HER2-negative early breast cancer by Swiss tumor boards using the 21gene recurrence score. BMC Cancer 17, 265 (2017).
27. Torres, S. et al. Prospective evaluation of the impact of the 21-gene Recurrence Score assay on adjuvant treatment decisions for women with node-positive breast cancer in Ontario, Canada. Oncologist 23, 768-775 (2018).

28. Ademuyiwa, F. O. et al. The effects of oncotype DX recurrence scores on chemotherapy utilization in a multi-institutional breast cancer cohort. Breast Cancer Res. Treat. 126, 797-802 (2011 Apr).

29. De Boer, R. H. et al. The impact of a genomic assay (Oncotype DX) on adjuvant treatment recommendations in early breast cancer. Med. J. Aust. 199, 205-208 (2013).

30. Lange, M. \& Joly, F. How to identify and manage cognitive dysfunction after breast cancer treatment. J. Oncol. Pr. 13, 784-790 (2017).

31. Petkov, V. I. et al. Breast-cancer-specific mortality in patients treated based on the 21-gene assay: a SEER population-based study. NPJ Breast Cancer 2, 16017 (2016); erratum 4, 17 (2018).

32. Peugniez, C. et al. Return to work after treatment for breast cancer: single center experience in a cohort of 273 patients. Bull. Cancer 98, E69-79 (2011).

33. Laas, E. et al. Evaluation of the costs and resource use associated with adjuvant chemotherapy for breast cancer in France. J. Med. Econ. 15, 1167-1175 (2012).

34. Wuerstlein, R. et al. Strong impact of MammaPrint and BluePrint on treatment decisions in luminal early breast cancer: results of the WSG-PRIMe study. Breast Cancer Res. Treat. 175, 389-399 (2019).

35. Exner, R. et al. The multigene signature MammaPrint impacts on multidisciplinary team decisions in ER+, HER2 - early breast cancer. Br. J. Cancer 111, 837-842 (2014).

36. Müller, B. M. et al. The EndoPredict gene-expression assay in clinical practice performance and impact on clinical decisions. PLOS ONE 8, e68252 (2013).

37. Wuerstlein, R. et al. The West German Study Group breast cancer intrinsic subtype study: a prospective multicenter decision impact study utilizing the Prosigna assay for adjuvant treatment decision-making in estrogen-receptor-positive, HER2negative early-stage breast cancer. Curr. Med. Res. Opin. 32, 1217-1224 (2016).

38. Hequet, D. et al. Prospective, multicenter French study evaluating the clinical impact of the Breast Cancer Intrinsic Subtype-Prosigna ${ }^{\circledR}$ Test in the management of early-stage breast cancers. PLOS ONE 12, e0185753 (2017)

39. Cognetti, F. et al. PONDx: real-life utilization and decision impact of the 21-gene assay on clinical practice: in Italy. figshare https://doi.org/10.6084/m9. figshare.13049816 (2020).

\section{ACKNOWLEDGEMENTS}

We thank the participating physicians and treatment centers for their contribution to this study. We also thank all patients for their willingness to participate. This work was supported by Genomic Health, Inc., by providing the Oncotype DX Breast Recurrence Score ${ }^{\circledast}$ test for the participating patients and by funding the Editorial assistance provided by Markus Fischer (Fischer BioMedical Wissenschaftskommunikation, Homburg/Saar, Germany).

\section{AUTHOR CONTRIBUTIONS}

Conception and design: F.C., R.M., A.F., G.N., and S.B. Provision of study materials or patients: all authors. Collection and assembly of data: F.C., R.M., A.F., G.N., and S.B. Data analysis and interpretation: F.C., R.M., A.F., G.N., and S.B. Manuscript writing/final approval: all authors.

\section{COMPETING INTERESTS}

F.C.: Genomic Health, Inc., Roche, Eli Lilly, Bayer, Novartis, Amgen, Pfizer, Astra Zeneca, Eisai, Merch-Serono, Boheringer Ingelheim, MSD, BMS, Takeda, Astellas Oncology, Abbott. S.B.: Genomic Health, Inc. A.F.: Roche, Celgene, Astra Zeneca, Eli Lilly, Novartis, Pfizer, Eisai. M.G.: Roche, Pfizer, Astra Zeneca, Novartis, Celgene, Eli, Lilly, Amgen, and Eisai. M.C.: Novartis, Pierre Fabre, Pfizer, OBI Pharma, Puma Biotechnology, Celldex Astra zeneca. A.Z.: Roche, Astra Zeneca, Novartis, Eli Lilly, Pfizer. D.G.: Eli Lilly, Novartis, Pfizer, Eisai. D.A.: Genomic Health, Inc. G.N.: Pfizer, Genomic Health, Inc., Italfarmaco. P.V.: Eisai, Roche, Pfizer, Novartis, Gentili. D.C.: Novartis, Roche. G.B.: Eli Lilly, Novartis. G.T.: Novartis Pfizer Italfarmaco e Molteni. T.G.: Novartis Roche Celgine Pfeizer Lilly Gentili. G.C., R.M., D.F., A.R., P.S., F.S., L. Vigna, C.T., D.S., M.A.F., E.M., G.S., G.P., A.F.S., R. B., and L. Vassalli declare no competing interests.

\section{ADDITIONAL INFORMATION}

Supplementary information The online version contains supplementary material available at https://doi.org/10.1038/s41523-021-00246-4.

Correspondence and requests for materials should be addressed to F.C. 
Reprints and permission information is available at http://www.nature.com/ reprints

Publisher's note Springer Nature remains neutral with regard to jurisdictional claims in published maps and institutional affiliations.

Open Access This article is licensed under a Creative Commons Attribution 4.0 International License, which permits use, sharing, adaptation, distribution and reproduction in any medium or format, as long as you give appropriate credit to the original author(s) and the source, provide a link to the Creative Commons license, and indicate if changes were made. The images or other third party material in this article are included in the article's Creative Commons license, unless indicated otherwise in a credit line to the material. If material is not included in the article's Creative Commons license and your intended use is not permitted by statutory regulation or exceeds the permitted use, you will need to obtain permission directly from the copyright holder. To view a copy of this license, visit http://creativecommons. org/licenses/by/4.0/.

(c) The Author(s) 2021 